\title{
Ultrafine Resveratrol Particles: Supercritical Antisolvent Preparation and Evaluation In Vitro and In Vivo
}

\author{
Kunlun Wang, Xiuhua Zhao, Yuangang Zu, Jialei Li, Xiaonan Zhang, \\ Wei Sun, and Xinyang Yu
}

Key Laboratory of Forest Plant Ecology, Northeast Forestry University, Ministry of Education, Hexing Road 26, Heilongjiang, Harbin 150040, China

Correspondence should be addressed to Xiuhua Zhao; xiuhuazhao@nefu.edu.cn and Yuangang Zu; yuangangzu@163.com

Received 9 January 2015; Accepted 5 March 2015

Academic Editor: Ying Li

Copyright ( 2015 Kunlun Wang et al. This is an open access article distributed under the Creative Commons Attribution License, which permits unrestricted use, distribution, and reproduction in any medium, provided the original work is properly cited.

Ultrafine resveratrol (u-Res) particles were prepared through the SAS process. The orthogonal method was used to optimize the factors of the SAS process. The size of $\mathrm{u}$-Res reached $0.68 \mu \mathrm{m}$ under the optimum conditions. The characterization of the $\mathrm{u}$-Res particles was tested by many analysis methods. The chemical structure of Res was unaffected by the SAS process. The degree of crystallinity of the u-Res particles greatly reduced. The purity of the u-Res particles increased from $98.5 \%$ to $99.2 \%$ during the SAS process. The u-Res particles had greater saturation solubility and dissolution rate than the raw-Res (r-Res) particles. The radical scavenging activity and bioavailability of the $\mathrm{u}$-Res in vivo were 1.9 times of the r-Res.

\section{Introduction}

Resveratrol (Res) (Figure 1) is a natural polyphenol [1] found in grapes [2]. Res in wine grapes reduces the risk of cardiovascular disease [3]. Res is found in 21 families, 31 genera, and 72 species of plants [4], such as peanuts [5], cocoa, and Polygonum cuspidatum [6]. Res is a natural health product that has potential use for treatment of several diseases because of its advantages such as the antitumor effect [7], antioxidant and free radical scavenging effect [8], antithrombotic effect [9], antibacterial effect [10], anti-inflammatory effect [11], prevention and treatment of neurological diseases [12], and enhancement of body immunity [13]. However, the poor water solubility and low bioavailability of Res greatly reduce its effect and application value [14]

In recent years, considerable efforts have been made to reduce the size of Res particles and encase water soluble materials on the surface of the Res particles through an antisolvent precipitation technology. The antisolvent precipitation technology increases the solubility of Res. Kim et al. were able to make the saturation solubility of Res reach 66.8 and $56.2 \mu \mathrm{g} / \mathrm{mL}$ in pure water and PBS solution $(\mathrm{pH}$ 7.4), respectively, which is much higher than the 31.8 and $29.6 \mu \mathrm{g} / \mathrm{mL}$ of $\mathrm{r}$-Res in the same condition. And they make
Res have a dissolution rate of $20 \%$ within 5 min and complete dissolution within $50 \mathrm{~min}$ [15]. However, this technology introduced other substances into Res; thus, high purity water soluble Res still remains unattainable.

The supercritical antisolvent (SAS) has been developed recently as a new nanopreparation technology $[16,17]$. The SAS process has several advantages over the other methods: the size of the product particle is micro, the biological activity of samples (especially thermal sensitive materials) is kept, it uses minimal residual solvent, and it is environment friendly $[18,19]$. The principle of the SAS process is that the $\mathrm{CO}_{2}$ gas becomes a supercritical fluid under high temperature and high pressure [20]. The sample organic solvent solution is then sprayed into the supercritical fluid vessel through the capillary nozzle by solution pump. When the sample organic solvent mixes with the supercritical fluid, the sample is recrystallized to precipitation. The process of recrystallization precipitation is so fast that mixed solutions have great supersaturation in only a short period. The grain size of recrystallization precipitation reaches the micro grade [21].

Because of the above advantages of SAS, the innovation of this paper aims to prepare u-Res particles to solve the poor water solubility problem of Res using SAS process. And using the method of SAS in preparing $\mathrm{u}$-Res particles is rarely 


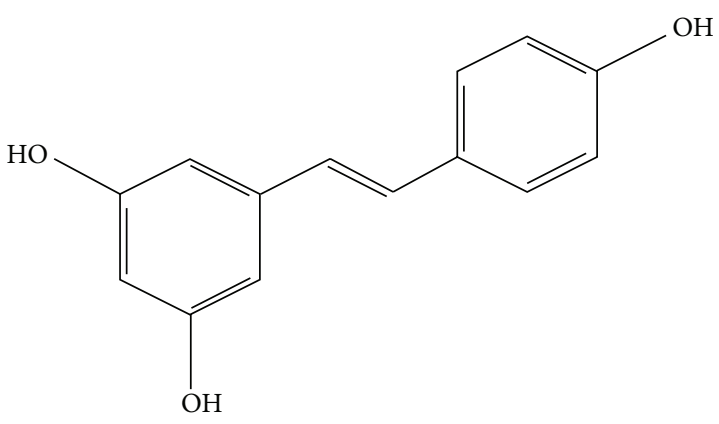

Figure 1: Chemical structure of Res (molecular formula: $\mathrm{C}_{14} \mathrm{H}_{12} \mathrm{O}_{3}$; molecular weight: 228.25).

reported. Then, the orthogonal method was used to optimize the factors of the SAS process to prepare u-Res particles under the best conditions. Moreover, the influences of characterization in u-Res particles were inspected by SEM, FTIR, LCMS, XRD, TG, DSC, HPLC analyses, saturation solubility, dissolution rate evaluation in vitro, and bioavailability in vivo. And the $\mathrm{u}$-Res particles are compared with the results of previous researchers to prove the superiority.

\section{Materials and Methods}

2.1. Materials. Res (mass purity $\geq 98.5 \%$ ) was purchased from Riotto Botanical Co., Ltd., China. Res standard substance (mass purity $\geq 99.9 \%$ ) was purchased from Selleck Biological Technology Co. Ltd., USA. $\mathrm{CO}_{2}$ (mass purity $\geq 99.9 \%$ ) was purchased from Harbin Liming Gas Co., Ltd., China. Ethanol (mass purity $\geq 99.7 \%$ ) was supplied by Tianjin Tianli Chemistry Co., Ltd., China. Methanol (mass purity $\geq 99.9 \%$ ) and acetonitrile (mass purity $\geq 99$. 9\%) were supplied by Beijing J\&KCHEMICA Co., Ltd., China. Hydrochloric acid (mass purity $\geq 38 \%$ ) was supplied by Tianjin Fuyu Fine Chemistry Co., Ltd., China. All materials were directly used without further purification.

2.2. Preparation of the $u$-Res Particle. Figure 2 illustrates the operating procedure of the SAS process apparatus. $\mathrm{CO}_{2}$ gas was cooled by a cooler (3) through a valve (2). The cooled $\mathrm{CO}_{2}$ gas was then compressed by $\mathrm{CO}_{2}$ pump (4) and continuously introduced into a heat exchanger (5), which cooled the $\mathrm{CO}_{2}$ pump at the same time. The $\mathrm{CO}_{2}$ gas was preheated in the heat exchanger (5) and then entered into a precipitation chamber (8). The $\mathrm{CO}_{2}$ was liquefied in the precipitation chamber (8) and completely filled a stainless steel frit vessel (7) through a $200 \mathrm{~nm}$ pore. Upon achieving a steady state, Res ethanol solution from a solution supply (13) was pumped using a solution pump (14) and sprayed into the stainless steel frit vessel (7) through a valve (15) and a capillary nozzle (6). When the Res ethanol solution mixed with the $\mathrm{CO}_{2}$ supercritical fluid, Res was quickly recrystallized to micron precipitation. Simultaneously, the $\mathrm{SC}-\mathrm{CO}_{2}$ /ethanol mixture passes through the $200 \mathrm{~nm}$ pore of the stainless steel frit vessel (7), and then entered into a gas-liquid separation vessel (10) through the valve (9); only the u-Res precipitation was left. In
TABLE 1: The factors and levels of the orthogonal array design.

\begin{tabular}{ccccc}
\hline Factor & $\begin{array}{c}A \\
\text { Temperature } \\
\left({ }^{\circ} \mathrm{C}\right)\end{array}$ & $\begin{array}{c}B \\
\text { Concentration } \\
(\mathrm{mg} / \mathrm{mL})\end{array}$ & $\begin{array}{c}C \\
\text { Pressure } \\
(\mathrm{MPa})\end{array}$ & $\begin{array}{c}D \\
\text { Flow rate } \\
(\mathrm{mL} / \mathrm{min})\end{array}$ \\
\hline Levels & & & & \\
1 & 40 & 1.0 & 10 & 1.0 \\
2 & 45 & 5.0 & 15 & 3.0 \\
3 & 50 & 9.0 & 20 & 5.0 \\
4 & 55 & 13.0 & 25 & 7.0 \\
\hline
\end{tabular}

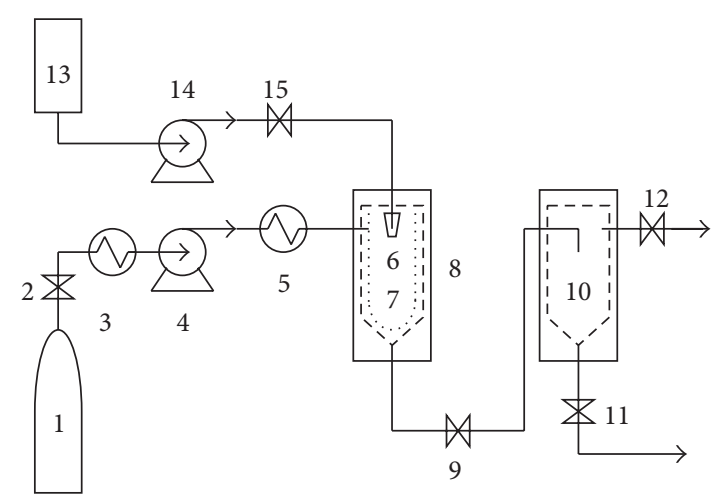

FIGURE 2: Flow diagram of the supercritical antisolvent (SAS) process. (1): $\mathrm{CO}_{2}$ cylinder; (2), (9), (11), (12), and (15): valves; (3): cooler; (4): $\mathrm{CO}_{2}$ pump; (5): heat exchanger; (6): capillary nozzle; (7): stainless steel frit vessel; (8): precipitation chamber; (10): gas-liquid separation vessel; (13): solution supply; (14): solution pump.

the as-liquid separation vessel (10), the $\mathrm{CO}_{2}$ supercritical fluid was gasified to enter the circulation through a valve (12), and the ethanol solution was recycled using a valve (11). Moreover, the $\mathrm{CO}_{2}$ pump (4) was stopped for $30 \mathrm{~min}$ when all of the Res ethanol solution was sprayed into the precipitation chamber (8); the steady state of the precipitation chamber (8) was maintained. The $\mathrm{CO}_{2}$ pump was then rebooted to keep the $\mathrm{CO}_{2}$ flowing for $30 \mathrm{~min}$ and remove the residual ethanol in $\mathrm{u}$-Res precipitation. Finally, the u-Res particles were collected for further characterization analysis.

2.3. Optimization of the SAS Process. An orthogonal design L16 $(4 \wedge 5)$ was selected to optimize the SAS process of the $\mathrm{u}$-Res particles (Table 1). The factors of the SAS process were pressure $(\mathrm{MPa})$, temperature $\left({ }^{\circ} \mathrm{C}\right)$, Res ethanol solution concentration $(\mathrm{mg} / \mathrm{mL})$, and sample solution flow rate $(\mathrm{mL} / \mathrm{min})$. Each factor level gained range through preliminary experiments. The particle size of the $\mathrm{u}$-Res particles was used as dependent variable. Data were analyzed using the orthogonal software assistant for optimum condition. Then the u-Res particles under this condition were obtained for characterization by various analytical methods.

\subsection{Particle Characterization}

2.4.1. Scanning Electron Microscopy (SEM). The surface morphologies of the Res samples were observed using scanning 
electronic microscopy (SEM) equipment (Quanta 200, FEI, USA). First, the r-Res and u-Res samples were glued on metal button by conducting resin and sprayed with a thin layer of gold on the surface. These metal buttons were then measured at a high voltage $(12.5 \mathrm{KV})$. Based on the SEM pictures, the last particle size of the Res samples was calculated using the Image-Pro Plus 6.0 software, providing data to support the further optimization of the SAS process.

2.4.2. Fourier Transform Infrared Spectroscopy (FT-IR). Spectra of $\mathrm{r}$-Res and $\mathrm{u}$-Res particles were collected for contrast using Fourier transform infrared (FT-IR) equipment (IRAffinity-1, Shimadzu, Japan). $2 \mathrm{mg}$ of r-Res and u-Res samples was mixed with $200 \mathrm{mg} \mathrm{KBr}$ and made to flake. Flaking was then measured in the wavenumber range from $500 \mathrm{~cm}^{-1}$ to $4000 \mathrm{~cm}^{-1}$.

\subsubsection{Liquid Chromatography-Mass Spectrometry (LC-MS).} Liquid chromatography-mass spectrometry (LC-MS) equipment (API300, MDS Sciex, USA) was used to obtain the spectra of the r-Res and u-Res particles. The detection condition of LC-MS was $100-1000 \mathrm{~m} / z$, the mobile phase was $50 \%(\mathrm{v} / \mathrm{v})$ methanol, the flow rate was $0.2 \mathrm{~mL} / \mathrm{min}$, and the sample solution concentration was $2.5 \mathrm{mg} / \mathrm{mL}$ [22]. Other conditions were in accordance with the 2.5 detection method.

2.4.4. X-Ray Diffraction (XRD). The crystallinity of the Res particles was analyzed using X-ray diffraction (XRD) equipment (XPert Pro MPD, Philips, Netherlands). The detection conditions were at room temperature, $\mathrm{Cu}$-ka radiation generated at $30 \mathrm{~mA}$ and $50 \mathrm{kV}$, scanned $3^{\circ}$ to $60^{\circ}$ ( $0.02^{\circ}$ per minute). Then, the $\mathrm{u}$-Res particles were placed 4 months under low temperature and less light conditions and analyzed again.

2.4.5. Differential Scanning Calorimetry (DSC). Differential scanning calorimetry (DSC) equipment (Q2000, TA, USA) was used to obtain the thermal behavior of the r-Res and $\mathrm{u}$-Res particles. The $3.0 \mathrm{mg}$ weight samples were placed in airtight aluminum pans and heated from $45^{\circ} \mathrm{C}$ to $300^{\circ} \mathrm{C}$ at a heating rate of $10^{\circ} \mathrm{C} / \mathrm{min}$.

2.4.6. Thermogravimetric Analysis (TGA). Thermogravimetric analysis of the Res samples was performed using a Perkin Elmer Pyris TGA (Perkin-Elmer Co., USA). The experiments were carried out with a heating rate of $1^{\circ} \mathrm{C} / \mathrm{min}$ under nitrogen flow of $50 \mathrm{~mL} / \mathrm{min} .5 .0 \mathrm{mg}$ of samples was placed in open aluminum pans, and a percentage weight loss from $32.5^{\circ} \mathrm{C}$ to $712.5^{\circ} \mathrm{C}$ was monitored.

2.5. Purity Detection. Res standard substance was prepared in different concentrations of aqueous solutions of the Res. These solutions were then detected using high performance liquid chromatography (HPLC) equipment (15252489, Watershed, USA) for linear regression equations.

The r-Res and $\mathrm{u}$-Res were, respectively, weighed to $2.0 \mathrm{mg}$ and dissolved under ultrasound in $3 \mathrm{~mL}$ of $95 \%$ ethanol for $30 \mathrm{~min}$. The solutions were then centrifuged at $10000 \mathrm{r} / \mathrm{min}$ for $5 \mathrm{~min}$. Finally, the supernatant was joined with the HPLC. The detection conditions were HPLC column $(\mathrm{C} 18,25 \mathrm{~cm})$; detection wavelength $(305 \mathrm{~nm})$; mobile phase, acetonitrile and $0.2 \%$ glacial acetic acid water solution, $30: 70(\mathrm{~V} / \mathrm{V})$; injection volume $(10 \mu \mathrm{L})$, and flow rate $(1 \mathrm{~min} / \mathrm{mL})$ [23].

2.6. Saturation Solubility. To determine the saturation solubility of the u-Res and r-Res particles, $1 \mathrm{~g}$ of $\mathrm{u}$-Res and $\mathrm{r}$-Res was added to $5 \mathrm{~mL}$ of pure water and $\mathrm{pH} 7.4$ phosphate buffer solution (PBS) to ensure excess samples. Each suspension liquid was then sealed and mixed at a speed of $75 \mathrm{rpm}$ in a $25^{\circ} \mathrm{C}$ water bath kettle for $48 \mathrm{~h}$. The solution was injected into HPLC through $0.22 \mu \mathrm{m}$ filter membrane for testing according to the 2.5 detection method.

2.7. Dissolution Test. Approximately $5 \mathrm{mg}$ of $\mathrm{u}$-Res and $5 \mathrm{mg}$ of r-Res particles were each placed in $500 \mathrm{~mL}$ of $0.2 \mathrm{~mol} / \mathrm{L}$ hydrochloric acid solution, with $0.5 \%(\mathrm{w} / \mathrm{w})$ Tween-80 at $37^{\circ} \mathrm{C}$ and a rotor speed of $75 \mathrm{rpm}$ [24]. Afterward, $1 \mathrm{~mL}$ of sample solution was transferred and taken through $0.22 \mu \mathrm{m}$ filter membrane into the HPLC at 1, 2, 3, 4, 5, 10, 15, and $20 \mathrm{~min}$. Finally, each sample solution was tested and repeated three times according to the 2.5 detection method.

2.8. DPPH Radical Scavenging Activity. The DPPH (1,1diphenyl-2-picrylhydrazyl radical 2,2-diphenyl-1-(2,4,6-trinitrophenyl) hydrazyl) researched radical scavenging activity of the u-Res and r-Res was used. First, different concentrations of $\mathrm{u}$-Res and $\mathrm{r}$-Res suspension water solutions $(3.125 \mu \mathrm{g} / \mathrm{mL}$ to $500 \mu \mathrm{g} / \mathrm{mL})$ were prepared. The solutions were then filtrated through the $0.45 \mu \mathrm{m}$ membrane and $1.5 \mathrm{~mL}$ solution of water was added to $1.5 \mathrm{~mL} 0.1 \mathrm{mmol} / \mathrm{L}$ DPPH 95\% ethanol solution, mixed, ultrasonically treated for $5 \mathrm{~min}$, and placed for $30 \mathrm{~min}$ at room temperature in the dark. Finally, the absorbencies of the solutions were measured at $517 \mathrm{~nm}$ by UV-detector. The computation formula of the $\mathrm{DPPH}(R \%)$ is as follows: $R \%=\left(1-A / A_{0}\right) \times 100 \%$, where $A_{0}$ is the absorbency of the blank sample and $A$ is the sample absorbency. Using the $\mathrm{IC}_{50}$ analysis software, $R \%$ of the sample of the different suspension concentrations of the water solutions was calculated for $\mathrm{IC}_{50}$ values. $\mathrm{IC}_{50}$ was the suspension concentration when sample scavenged half of the free radical.

2.9. Bioavailability Studies. Six female Sprague-Dawley rats (weight between 200 and $250 \mathrm{~g}$, age between 60 and $70 \mathrm{~d}$ ) were prepared and divided into two groups, each group of 3 rats. Prior to all experiments, animals were fasted overnight and freely drunk water. Two groups of rats were, respectively, given $\mathrm{r}$-Res and $\mathrm{u}$-Res by gavage at the doses of $50 \mathrm{mg} / \mathrm{kg}$ (according to Res). After feeding, blood samples from the two groups were obtained at $0.25,0.5,1,2,3,4,6$, and $8 \mathrm{~h}$. The blood samples were then centrifuged at $10000 \mathrm{rpm}$ for $10 \mathrm{~min}$. The supernatants of the blood samples were mixed with methanol 1:3(v/v) and centrifuged at $10000 \mathrm{rpm}$ for $10 \mathrm{~min}$. The supernatants of the mixed solutions were measured according to the 2.5 detection method. Experimental procedures and animal use and care protocols were approved 
TABLE 2: Orthogonal array design OA16 $(4)^{5}$ and experimental results.

\begin{tabular}{|c|c|c|c|c|c|}
\hline Trial number & $A$ & $B$ & $C$ & $D$ & $\operatorname{MPS}(\mu \mathrm{m}) \pm \mathrm{SD}(n=3)$ \\
\hline 1 & 1 & 1 & 1 & 1 & $1.79 \pm 0.12$ \\
\hline 2 & 1 & 2 & 2 & 2 & $1.89 \pm 0.18$ \\
\hline 3 & 1 & 3 & 3 & 3 & $0.82 \pm 0.04$ \\
\hline 4 & 1 & 4 & 4 & 4 & $1.31 \pm 0.09$ \\
\hline 5 & 2 & 1 & 2 & 3 & $2.18 \pm 0.24$ \\
\hline 6 & 2 & 2 & 1 & 4 & $1.49 \pm 0.16$ \\
\hline 7 & 2 & 3 & 4 & 1 & $1.03 \pm 0.08$ \\
\hline 8 & 2 & 4 & 3 & 2 & $1.09 \pm 0.05$ \\
\hline 9 & 3 & 1 & 3 & 4 & $0.76 \pm 0.04$ \\
\hline 10 & 3 & 2 & 4 & 3 & $1.44 \pm 0.12$ \\
\hline 11 & 3 & 3 & 1 & 2 & $0.82 \pm 0.08$ \\
\hline 12 & 3 & 4 & 2 & 1 & $0.7 \pm 0.02$ \\
\hline 13 & 4 & 1 & 4 & 2 & $0.93 \pm 0.04$ \\
\hline 14 & 4 & 2 & 3 & 1 & $0.93 \pm 0.10$ \\
\hline 15 & 4 & 3 & 2 & 4 & $1.61 \pm 0.12$ \\
\hline 16 & 4 & 4 & 1 & 3 & $1.27 \pm 0.12$ \\
\hline$K_{1}{ }^{a}$ & $1.45 \pm 0.13$ & $1.42 \pm 0.12$ & $1.34 \pm 0.08$ & $1.11 \pm 0.06$ & \\
\hline$K_{2}$ & $1.45 \pm 0.14$ & $1.44 \pm 0.10$ & $1.60 \pm 0.09$ & $1.18 \pm 0.12$ & \\
\hline$K_{3}$ & $0.93 \pm 0.07$ & $1.07 \pm 0.06$ & $0.90 \pm 0.08$ & $1.43 \pm 0.14$ & \\
\hline$K_{4}$ & $1.19 \pm 0.10$ & $1.09 \pm 0.12$ & $1.18 \pm 0.13$ & $1.29 \pm 0.08$ & \\
\hline$R^{\mathrm{b}}$ & 0.52 & 0.37 & 0.70 & 0.32 & \\
\hline Optimal level & $A_{3}$ & $B_{3}$ & $C_{3}$ & $D_{1}$ & \\
\hline
\end{tabular}

by the Committee on Ethical Use of Animals of Harbin Medical University.

\section{Results and Discussion}

3.1. Optimization Studies. The SEM images for $\mathrm{u}-\mathrm{Res}$ particle size data, which the orthogonal assistant analyzed in Table 2, were measured using Image-Pro Plus 6.0. Table 2 shows that the minimum particle size of the $\mathrm{u}$-Res is $0.7 \pm 0.02 \mu \mathrm{m}$ and the maximum particle size is $2.18 \pm 0.24 \mu \mathrm{m}$. According to the $R$ value from Table 2, $C$ is the most important factor; the influencing factors of particle size is as follows: $C>$ $A>B>D$. In Figure 3, the trend of the particle size is erratic, rising then declining and then rising again under the conditions $A, B$, and $C$, with $A_{3}, B_{3}$, and $C_{3}$ as the lowest points. The trend of particle size initially increased and then decreased under condition $D$, and $D 1$ is the lowest point. Moreover, the fluctuation intensity is $C>A>B>D$ (i.e., pressure factor $>$ temperature factor $>$ concentration factor $>$ flow rate factor); pressure is the most important factor. The optimum condition of the SAS process of the u-Res particles is $A_{3} B_{3} C_{3} D_{1}$ (precipitation temperature at $50^{\circ} \mathrm{C}$, concentration at $9 \mathrm{mg} / \mathrm{mL}$, precipitation pressure at $20 \mathrm{MPa}$, and flow rate at $1 \mathrm{~mL} / \mathrm{min}$ ) according to the $k$ value from Table 2 and Figure 3. Then the u-Res particles was obtained under the optimum condition of $A_{3} B_{3} C_{3} D_{1}$ and the particle size of the confirmatory test is $0.68 \pm 0.03 \mu \mathrm{m}$. Then, the $\mathrm{u}$-Res particles under this condition were characterized by various analytical methods.

3.2. Morphology. Compared with the crystal column form of r-Res in Figure 4(a), the lamellar form of the u-Res particles is easily distinguishable in Figure 4(b). The u-Res particle size is smaller, generally, less than $2 \mu \mathrm{m}$, its crystal degree is lower, and its specific surface area is larger. According to the NoyesWhitney equation, the water solubility of the $\mathrm{u}$-Res particles is higher.

3.3. Purity Study. Figure $\mathrm{S}_{1}$ (see Figure $\mathrm{S}_{1}$ in the Supplementary Material available online at http://dx.doi.org/10.1155/ 2015/838513) shows the HPLC chromatograms of the r-Res and $\mathrm{u}$-Res particles in vitro. A standard curve of Res is drawn using HPLC, and the regressive equation is $y=$ $88905985.4722 x-48305.5021$ with $R^{2}=0.995$. The peak values of the r-Res and micro particles are placed into regressive equation for the contents. The contents of the rRes and micro particles are at $98.5 \% \pm 0.5$ and $99.2 \% \pm 0.5$ (mean $\pm \mathrm{SD}$ ), confirming that the Res is purified in the SAS process. The reason is that similar Res molecules crystallize to each other and reject other molecules in SAS process. Impurity is separated from Res. So, the purity of $\mathrm{u}$-Res after recrystallization by SAS process is higher than $\mathrm{r}$-Res. 


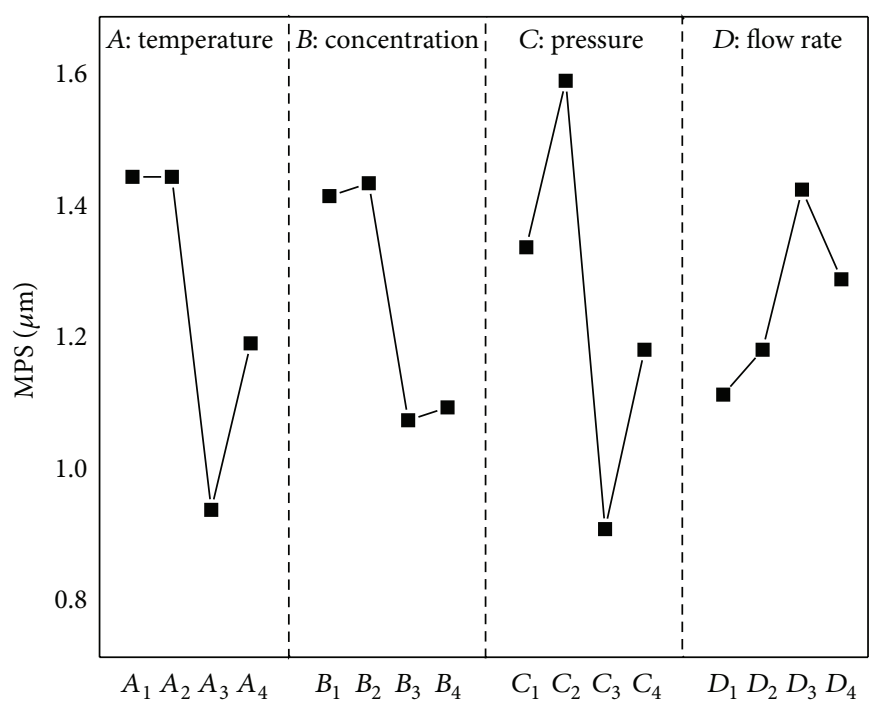

Figure 3: The effect of each parameter on the particles size of $u$-Res ( $A$ is temperature, $B$ is concentration, $C$ is pressure, and $D$ is flow rate).

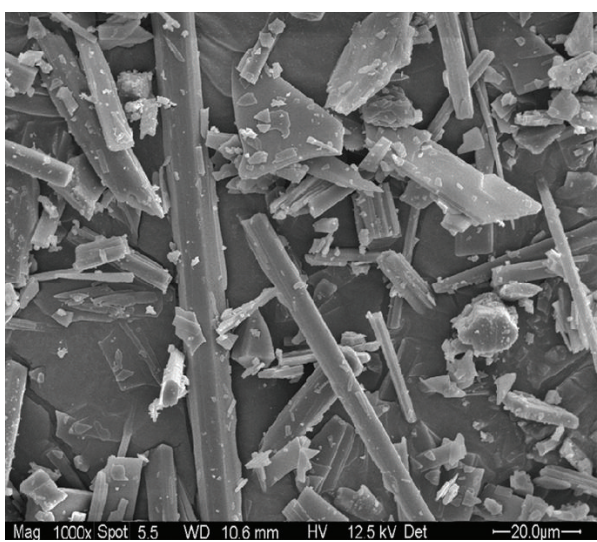

(a)

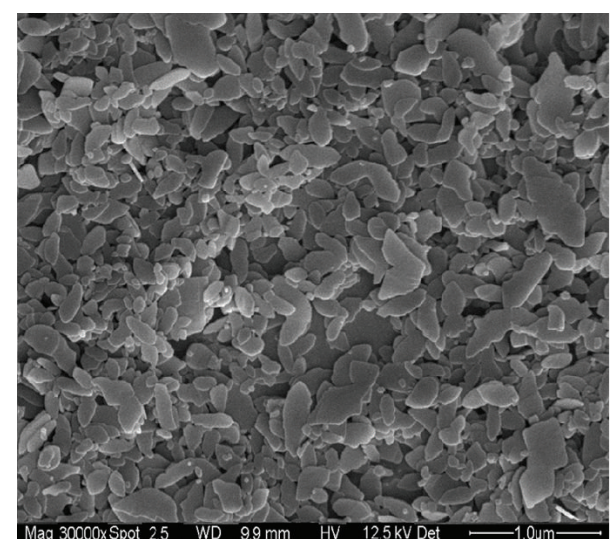

(b)

FIGURE 4: Scanning electron micrographs of r-Res (a) and u-Res (b).

\subsection{Chemical Structure Studies}

3.4.1. FT-IR Spectroscopy. The molecular structures of the rRes and $\mathrm{u}$-Res particles were examined using FT-IR equipment. Figure 5 shows no significant differences between the two particles in 3331, 3014, 1888, 1593, 1512, 1328, 1149, 964, 833, 675 , and $518 \mathrm{~cm}^{-1}$. Therefore, the functional groups of the $\mathrm{r}-$ Res and micro particles did not exhibit particular changes in the SAS process.

3.4.2. Liquid Chromatography-Mass Spectrometry (LC-MS). The molecular weights of the $\mathrm{r}$-Res and $\mathrm{u}$-Res particles were 226.8 and 227.0, as shown in Figure 6. Results of the LC-MS clearly showed that the two particles have the same molecular weight, and the molecular weight is the same as Res at 226.8 [24], indicating that the Res is accurate. Res did not exhibit any change in the SAS process.

\subsection{Physical Structure Studies}

3.5.1. X-Ray Diffraction Studies. Figure 7 shows that the XRD pattern of the $r$-Res has a high-intensity diffraction peak at $2 \theta=6.6^{\circ}, 16.3^{\circ}$, and $19.1^{\circ}$. Although the u-Res particles have diffraction peaks at the same position as the XRD pattern, the intensity of the diffraction peaks of micro particles is far below the level of the raw particles. Results suggest that the crystallization degree of Res is significantly reduced in the SAS process; thus, improving the dissolution of Res in water can be beneficial. Figure 7 also shows that the XRD pattern of $\mathrm{u}$-Res particles which was placed 4 months under low temperature has no change. This proves that the $\mathrm{u}$-Res is stable.

3.5.2. Differential Scanning Calorimetry. Compared with the DSC patterns of the r-Res and u-Res particles in Figure 8, two similar sharp endothermic peaks were found at $267^{\circ} \mathrm{C}$ and $265^{\circ} \mathrm{C}$. The peak of the u-Res particles is far below the 


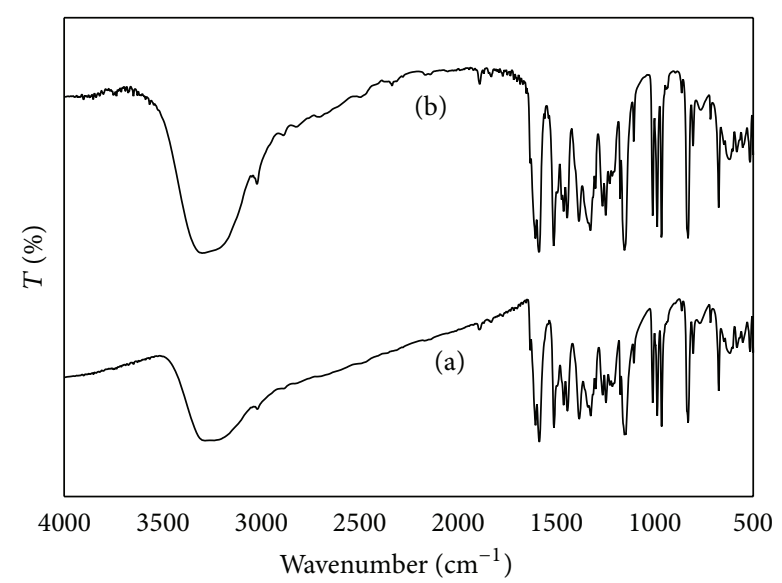

FIGURE 5: FTIR spectra of r-Res (a) and u-Res (b).

r-Res. This illustrated that the crystallization degree of $\mathrm{u}$ Res is significantly reduced and is beneficial to improve the solubility and the bioavailability [25].

3.5.3. Thermogravimetric Analysis. Figure 9 shows that the TG curves of the r-Res and u-Res particles both have a slow weight drop below $260^{\circ} \mathrm{C}$ and a sudden weight drop between 260 and $712^{\circ} \mathrm{C}$, indicating that both $\mathrm{r}$-Res and $\mathrm{u}$-Res have small water and volatile constituents to lose below $260^{\circ} \mathrm{C}$. And the melting point of Res is $253-255^{\circ} \mathrm{C}$. So the Res begins to decompose with temperature increasing at $260^{\circ} \mathrm{C}$ [26]. Figure 9 also shows the total weight loss of $r$-Res and u-Res is $50 \%$ and $53.5 \%$, respectively, which indicates that $\mathrm{u}-\mathrm{Res}$ has more weight loss than $r$-Res between 260 and $712^{\circ} \mathrm{C}$. The reason is that the smaller the particle size is, the bigger the specific surface area is, the more the atoms are on the surface of particles, the more its surface energy will increase quickly, the higher the specific surface energy is, the worse the stability of the particles is, and the more the weight loss is at high temperatures [27]. So the u-Res particles are smaller in size and lead to a high specific surface energy, which explains why the $\mathrm{u}$-Res has more weight loss than r-Res.

3.6. Saturation Solubility and Dissolution Rate. Figure 10 shows that saturation solubility of $\mathrm{u}-\operatorname{Res}(75.1 \mu \mathrm{g} / \mathrm{mL})$ in pure water is much higher than that of $\mathrm{r}-\operatorname{Res}(31.8 \mu \mathrm{g} / \mathrm{mL})$. The saturation solubility of $\mathrm{u}$-Res $(66.2 \mu \mathrm{g} / \mathrm{mL})$ in PBS solution is much higher than the r-Res $(29.6 \mu \mathrm{g} / \mathrm{mL})(\mathrm{pH} 7.4)$. Thus, the saturation solubility of the u-Res is 2.4 times and 2.3 times of the r-Res in pure water and in PBS solution ( $\mathrm{pH} 7.4$ ). The solubility of r-Res and u-Res in pure water is slightly higher than in PBS solution ( $\mathrm{pH}$ 7.4). Combined with the study of the physical structure of Res, this indicates that the saturation solubility of Res increases with the decrease of the particle size. The Ostwald-Freundlich equation gives the principle of the above conclusion. The Ostwald-Freundlich equation is defined as follows:

$$
1 g \frac{S_{2}}{S_{1}}=\frac{2 \sigma M}{\rho R T}\left(\frac{1}{R_{2}}-\frac{1}{R_{1}}\right),
$$

where $R_{1}$ and $R_{2}$ are the particle radius; $S_{1}$ and $S_{2}$ are the solubility when the particle radius was $R_{1}$ and $R_{2} ; \rho$ is the density of solid drugs; $\sigma$ is the interfacial tension between solid drugs and liquid solvents; $M$ is molecular weight of the drug; $R$ is molar gas constant; and $T$ is thermodynamic temperature [28]. When the $R_{2}$ of $\mathrm{u}$-Res is smaller than the $R_{1}$ of $\mathrm{r}$-Res, the $S_{2}$ of $\mathrm{u}$-Res is greater than the $S_{1}$ of $\mathrm{r}$-Res. So the saturation solubility of Res increases with the decrease of the particle size. Kim et al. were able to make the saturation solubility of Res reach 66.8 and $56.2 \mu \mathrm{g} / \mathrm{mL}$ in pure water and PBS solution ( $\mathrm{pH} 7.4$ ), respectively [15]. So, compared with the results obtained by Kim et al., the u-Res prepared using the SAS process is shown to have more saturation solubility and does not contain any subsidiary material.

Figure 11 shows that the $\mathrm{u}$-Res particles have a dissolution rate of $89.5 \%$ within $5 \mathrm{~min}$ and complete dissolution within $15 \mathrm{~min}$, whereas the r-Res particles have a dissolution rate of only $72.6 \%$ at $5 \mathrm{~min}, 77.3 \%$ at $15 \mathrm{~min}$, and without completing dissolution $77.8 \%$ at $30 \mathrm{~min}$. The results clearly indicate that uRes particles have a much faster dissolution rate than $r$-Res. According to the Noyes-Whitney equation, the main factors that affect the sample dissolution rate are the dissolution interface area, sample saturation concentration, and sample solution concentration constant $k$ [29]. The Noyes-Whitney equations are defined as follows:

$$
\frac{d c}{d t}=k S(C s-C), \quad k=\frac{D}{V h},
$$

where $d c / d t$ is dissolution rate; $S$ is dissolution interface area; $C s$ is sample saturation concentration; $C$ is sample solution concentration; $D$ is diffusion coefficients; $V$ is dissolution medium quality; $h$ is boundary layer thickness. The sample dissolution rate is directly proportional to the interface area with dissolution medium [30]; as the interface area increases, the dissolution rate also increases. Decreased particle size leads to an increase in the effective surface area in the diffusion layer, thereby increasing the sample dissolution rate [31]. When the sample particle size decreases to less than $5 \mu \mathrm{m}$, the boundary layer thickness of the Prandtl boundary layer equation is reduced [32]. The constant $k$ will then be increased by the equation $k=D / V h$, substituted in the equation of $d c / d t=k S(C s-C)$; the sample dissolution rate increases.

As shown in Figure 4, the u-Res particles constitute tiny lamellar particles, whereas the r-Res particles constituted large crystal columns. So the u-Res particles are smaller than the $\mathrm{r}$-Res particles. The u-Res particles have a much larger interface area with dissolution medium and faster dissolution rate than the r-Res particles. According to Figure 12, the dissolution rate of the $\mathrm{u}$-Res is 1.3 times that of the r-Res. Kim et al. make Res have a dissolution rate of $20 \%$ within $5 \mathrm{~min}$ and complete dissolution within 50 min [15]. Compared with the results of Kim et al., the u-Res prepared by the SAS process has higher dissolution rate without any subsidiary material.

3.7. Radical Scavenging Activities. Six suspension concentration water solutions of $\mathrm{r}$-Res and $\mathrm{u}$-Res particles were tested through PDDH. As shown in Figure 12, the free radical 


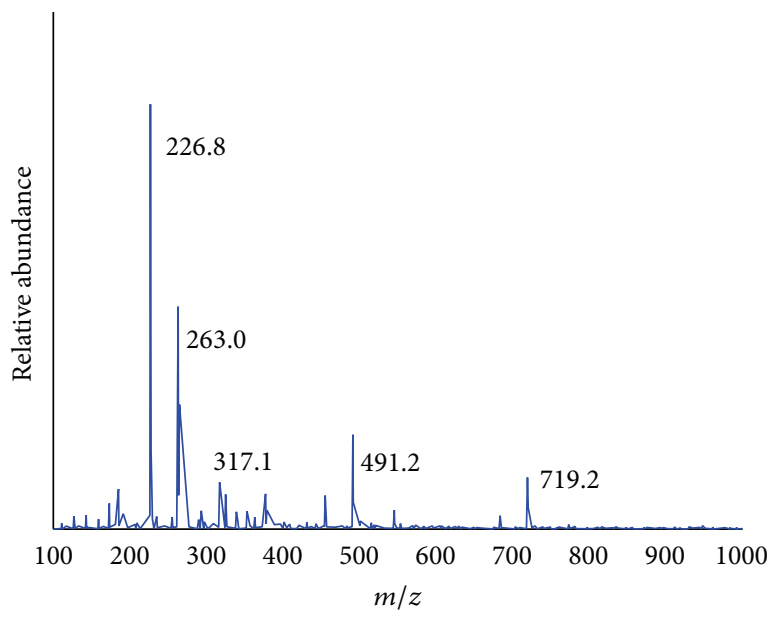

(a)

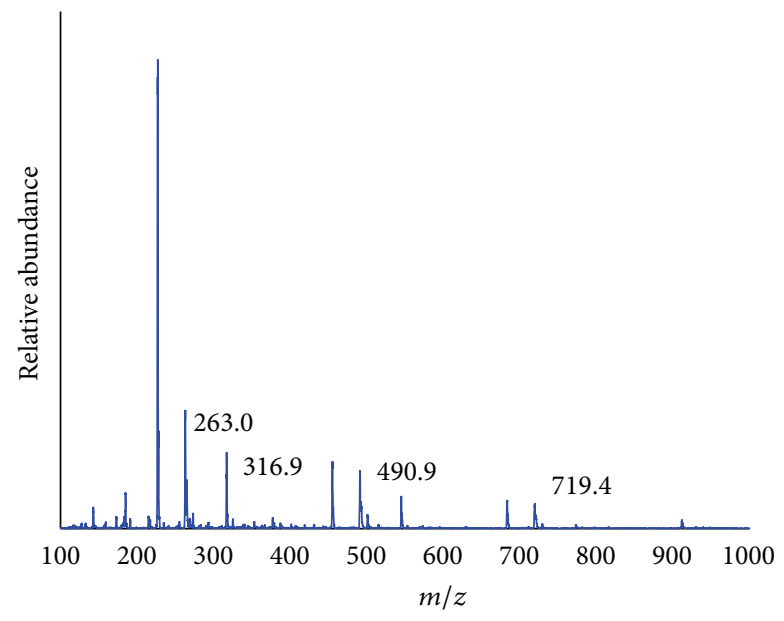

(b)

FiguRE 6: LC-MS spectra of r-Res (a) and u-Res (b).

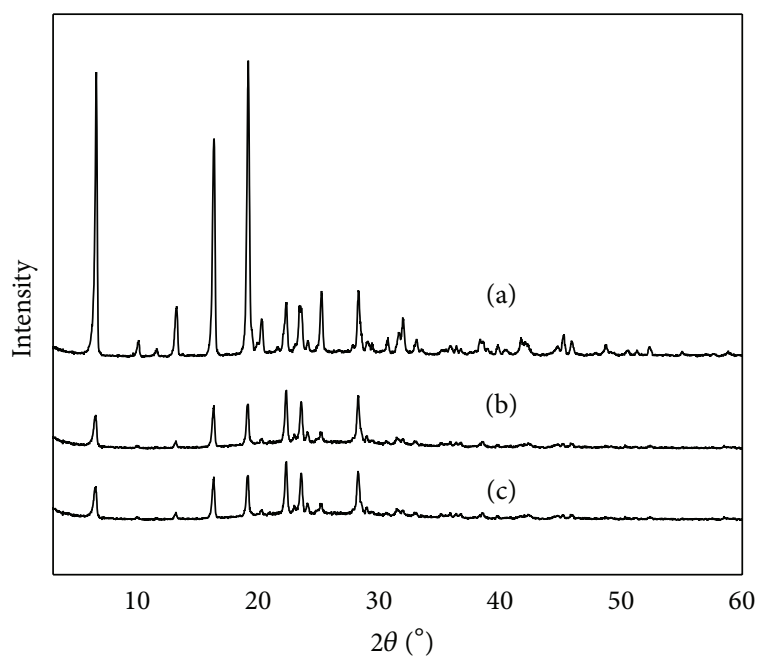

Figure 7: XRD patterns of r-Res (a), u-Res (b), and u-Res (c) which was placed 3 months.

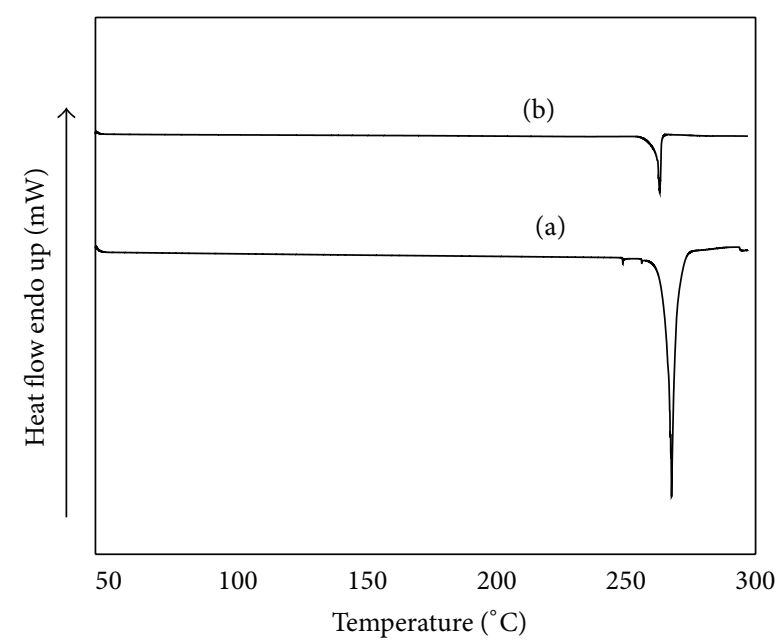

Figure 8: DSC curves of r-Res (a) and u-Res (b).

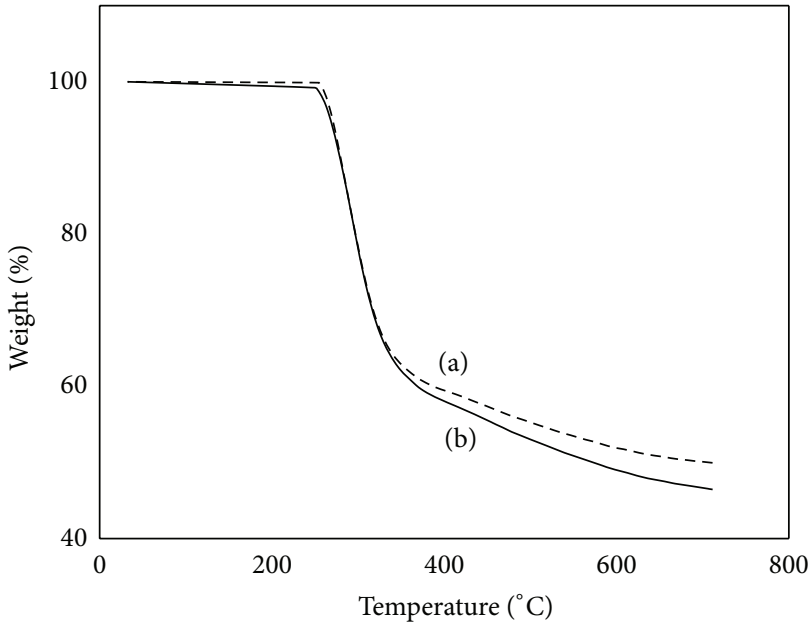

FIGURE 9: TG curves of r-Res (a) and u-Res (b).

scavenging rate of the $\mathrm{u}$-Res particles is much higher than the r-Res particles. Using the $\mathrm{IC}_{50}$ calculation software, the $\mathrm{IC}_{50}$ value of $\mathrm{r}$-Res $(14.55 \mu \mathrm{g} / \mathrm{mL})$ was much greater than the $\mathrm{u}$ Res particles $(7.75 \mu \mathrm{g} / \mathrm{mL})$, indicating that the $\mathrm{u}$-Res particles only needed half of the $r$-Res concentration to reach the same effect. The radical scavenging activity of the $\mathrm{u}$-Res is 1.9 times that of $\mathrm{r}$-Res. The dissolution rate of the $\mathrm{u}$-Res particles was larger than the r-Res according to Figure 12. Therefore, the $\mathrm{u}$-Res particles greatly improved the free radical scavenging activity as a natural product and have potential in the health care products market.

3.8. Bioavailability Analysis. As shown in Figure 13, the result of bioavailability is the drug concentration of the u-Res is higher than the $\mathrm{r}$-Res in rat plasma. The drug concentration of the $\mathrm{u}$-Res reached the maximum of $2.35 \mu \mathrm{g} / \mathrm{mL}$ at $15 \mathrm{~min}$. Then the drug concentration of the u-Res particles decreased 


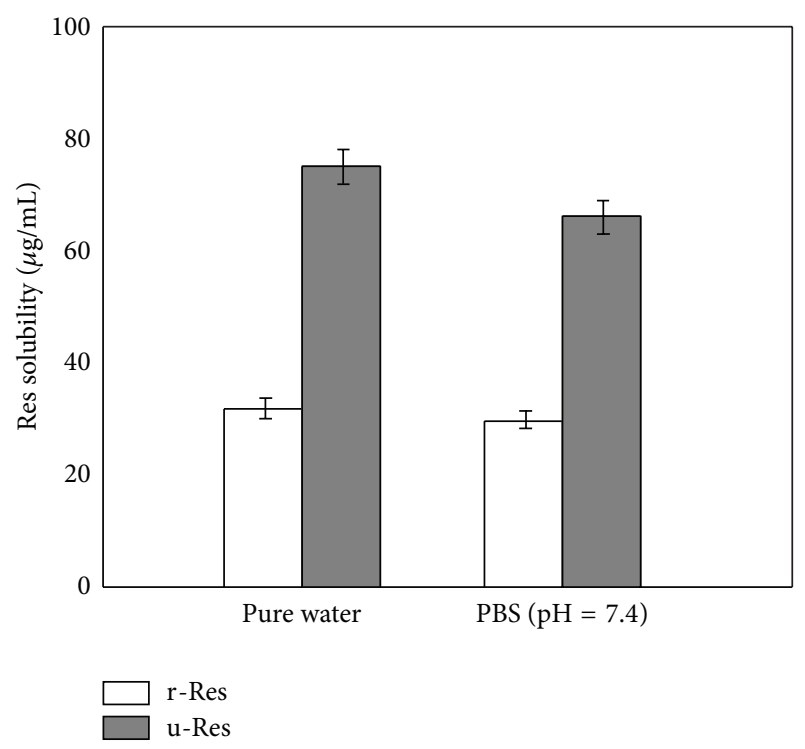

FIGURE 10: Saturation solubility of r-Res and u-Res in pure water and PBS solution ( $\mathrm{pH}$ 7.4).

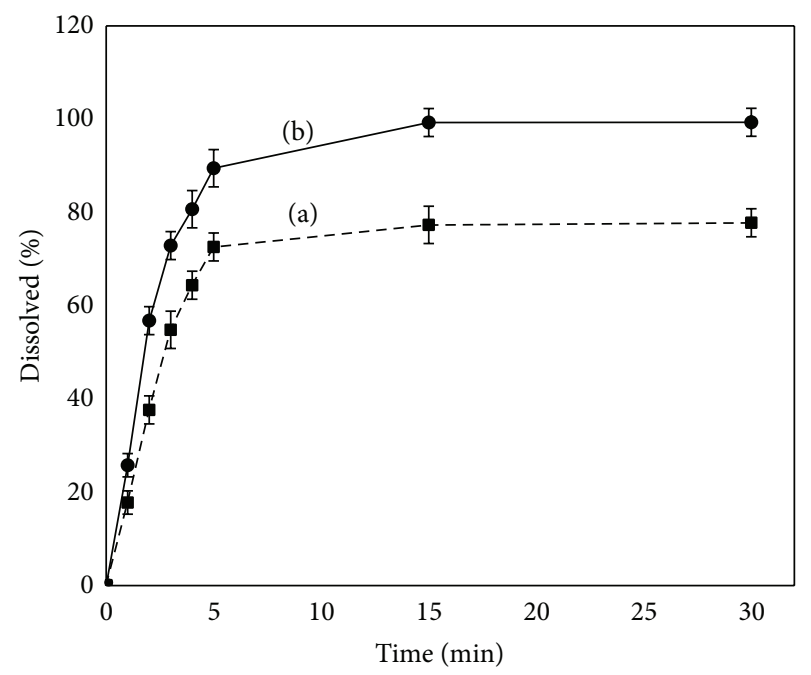

FIgURE 11: Solubility curves of r-Res (a) and u-Res (b) in vitro.

slowly to $0.01 \mu \mathrm{g} / \mathrm{mL}$ in $8 \mathrm{~h}$. The drug concentration of the rRes also reached the maximum of $1.95 \mu \mathrm{g} / \mathrm{mL}$ at $15 \mathrm{~min}$. Then, the drug concentration of the r-Res decreased to $0.01 \mu \mathrm{g} / \mathrm{mL}$ in $8 \mathrm{~h}$. Through the OriginLab 8.5 software, comparing the AUC of the two groups, the $\mathrm{u}-\mathrm{Res}$ is 1.9 times of the r-Res. The reason of the result is that the $\mathrm{u}$-Res has smaller particle size than $\mathrm{r}$-Res, which leads to the u-Res having larger interface area and higher saturation solubility. So, the SAS method improves the bioavailability of the Res.

\section{Conclusions}

This paper was conducted to make Res reach micron level through the SAS process and to optimize the factors of the SAS process by orthogonal method. Using SEM,

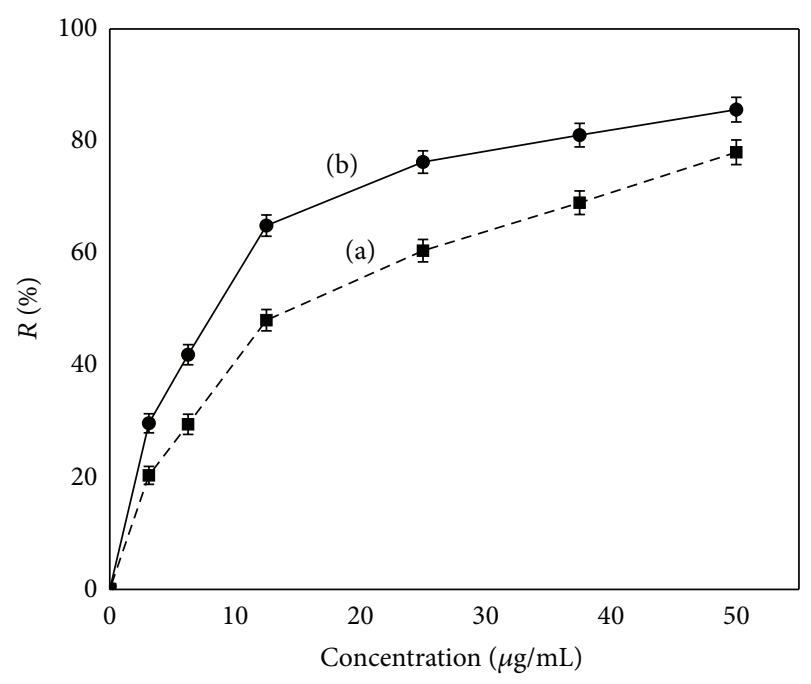

FIGURE 12: Percentage of free radical scavenging activity of r-Res (a) and $\mathrm{u}$-Res (b).

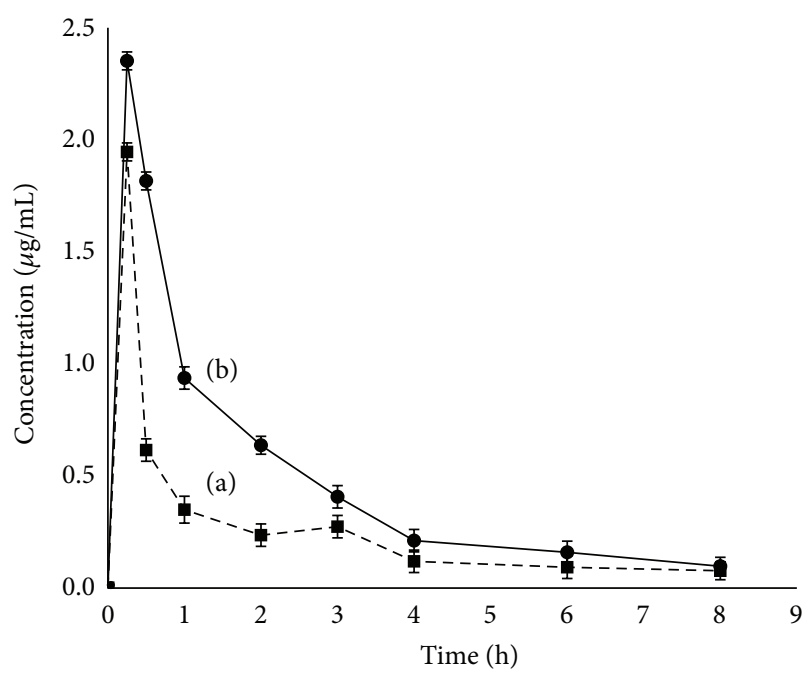

FIGURE 13: Plasma concentration versus time curve of r-Res (a) and u-Res (b).

the microstructure of Res was observed to have fundamentally changed from the large crystal column of r-Res to the tiny lamellar particle of u-Res under the SAS process. The average particle size was also reduced from hundreds of microns of r-Res to $0.68 \mu \mathrm{m}$ of u-Res. u-Res has the same chemical structure and physical structure as r-Res according to the FTIR, XRD, DSC, and TG data, indicating that the crystal degree of Res was greatly reduced and the structure did not change during the SAS process. Moreover, the SAS process purified the HPLC-detected Res from $98.5 \%$ to $99.2 \%$. Finally, a comparison between the r-Res and u-Res particles through saturation solubility studies, dissolution rate test in vitro, radical scavenging activity, and bioavailability test in vivo showed that the saturation solubility of the $\mathrm{u}$-Res was 2.4 times and 2.3 times of the r-Res in pure water and PBS solution ( $\mathrm{pH}$ 7.4). Moreover, Kim et al. reported that 
the dissolution rate of the u-Res was much faster than the Res. The radical scavenging activity and bioavailability of the $\mathrm{u}$-Res in vivo were 1.9 times of the $\mathrm{r}$-Res. Therefore, $\mathrm{u}$ Res particles made by the SAS process greatly increased the application value of Res particles as a new material.

\section{Conflict of Interests}

The authors declare that there is no conflict of interests regarding the publication of this paper.

\section{Acknowledgments}

The authors would like to acknowledge the financial support from the Special Fund for Forestry Scientific Research in the Public Interest (20140470102) and the National Natural Science Foundation of China (no. 21203018).

\section{References}

[1] A. R. Neves, M. Lúcio, Y. Zu, J. L. C. Lima, L. Zhang, and S. Reis, "Resveratrol in medicinal chemistry: a critical review of its pharmacokinetics, drug-delivery, and membrane interactions," Current Medicinal Chemistry, vol. 19, no. 11, pp. 1663-1681, 2012.

[2] P. Langcake and R. J. Pryce, “The production of resveratrol by Vitis vinifera and other members of the Vitaceae as a response to infection or injury," Physiological Plant Pathology, vol. 9, no. 1, pp. 77-86, 1976.

[3] T.-K. Lin, L.-T. Huang, Y.-H. Huang, M.-M. Tiao, K.-S. Tang, and C.-W. Liou, "The effect of the red wine polyphenol resveratrol on a rat model of biliary obstructed cholestasis: involvement of anti-apoptotic signalling, mitochondrial biogenesis and the induction of autophagy," Apoptosis, vol. 17, no. 8, pp. 871-879, 2012.

[4] C. Counet, D. Callemien, and S. Collin, "Chocolate and cocoa: new sources of trans-resveratrol and trans-piceid," Food Chemistry, vol. 98, no. 4, pp. 649-657, 2006.

[5] S. S. Lee, S. M. Lee, M. Kim, J. Chun, Y. K. Cheong, and J. Lee, "Analysis of trans-resveratrol in peanuts and peanut butters consumed in Korea," Food Research International, vol. 37, no. 3, pp. 247-251, 2004.

[6] B. Shan, Y.-Z. Cai, J. D. Brooks, and H. Corke, "Antibacterial properties of Polygonum cuspidatum roots and their major bioactive constituents," Food Chemistry, vol. 109, no. 3, pp. 530537,2008

[7] K.-H. Jung, J. H. Lee, C. H. T. Quach et al., "Resveratrol suppresses cancer cell glucose uptake by targeting reactive oxygen species-mediated hypoxia-inducible factor-lalpha activation," Journal of Nuclear Medicine, vol. 54, no. 12, pp. 2161-2167, 2013.

[8] K.-T. Lu, M.-C. Ko, B.-Y. Chen et al., "Neuroprotective effects of resveratrol on MPTP-induced neuron loss mediated by free radical scavenging," Journal of Agricultural and Food Chemistry, vol. 56, no. 16, pp. 6910-6913, 2008.

[9] S. Bae, E.-M. Lee, H. J. Cha et al., "Resveratrol alters microRNA expression profiles in A549 human non-small cell lung cancer cells," Molecules and Cells, vol. 32, no. 3, pp. 243-249, 2011.

[10] V. Katalinić, S. S. Možina, D. Skroza et al., "Polyphenolic profile, antioxidant properties and antimicrobial activity of grape skin extracts of 14 Vitis vinifera varieties grown in Dalmatia (Croatia)," Food Chemistry, vol. 119, no. 2, pp. 715-723, 2010.
[11] A. S. Yar, S. Menevse, E. Alp, F. Helvacioglu, and G. Take, “The effects of resveratrol on cyclooxygenase- 1 and cyclooxygenase2 mRNA and protein levels in diabetic rat kidneys," Molecular Biology Reports, vol. 37, no. 5, pp. 2323-2331, 2010.

[12] C. Dani, L. S. Oliboni, F. Agostini et al., "Phenolic content of grapevine leaves (Vitis labrusca var. Bordo) and its neuroprotective effect against peroxide damage," Toxicology in Vitro, vol. 24, no. 1, pp. 148-153, 2010.

[13] S. T. Ahmed, M. E. Hossain, G. M. Kim, J. A. Hwang, H. Ji, and C. J. Yang, "Effects of resveratrol and essential oils on growth performance, immunity, digestibility and fecal microbial shedding in challenged piglets," Asian-Australasian Journal of Animal Sciences, vol. 26, no. 5, pp. 683-690, 2013.

[14] H. Nemoto, A. Katagiri, M. Kamiya et al., "Synthesis and evaluation of water-soluble resveratrol and piceatannol via BGLation," Bioorganic \& Medicinal Chemistry Letters, vol. 22, no. 15 , pp. 5051-5054, 2012.

[15] S. Kim, W. K. Ng, Y. Dong, S. Das, and R. B. H. Tan, "Preparation and physicochemical characterization of transresveratrol nanoparticles by temperature-controlled antisolvent precipitation," Journal of Food Engineering, vol. 108, no. 1, pp. 3742, 2012.

[16] Y. B. Jiang, W. L. Sun, and W. Wang, "Recrystallization and micronization of 10-hydroxycamptothecin by supercritical antisolvent process," Industrial \& Engineering Chemistry Research, vol. 51, no. 6, pp. 2596-2602, 2012.

[17] A. Miguel, A. Martín, T. Gamse, and M. J. Cocero, "Supercritical anti solvent precipitation of lycopene: effect of the operating parameters," Journal of Supercritical Fluids, vol. 36, no. 3, pp. 225-235, 2006.

[18] X. H. Zhao, X. Q. Chen, Y. G. Zu, R. Jiang, and D. M. Zhao, "Recrystallization and micronization of taxol using the supercritical antisolvent (SAS) process," Industrial and Engineering Chemistry Research, vol. 51, no. 28, pp. 9591-9597, 2012.

[19] X. Zhao, Y. Zu, Q. Li et al., "Preparation and characterization of camptothecin powder micronized by a supercritical antisolvent (SAS) process," The Journal of Supercritical Fluids, vol. 51, no. 3, pp. 412-419, 2010.

[20] M.-S. Kim, S. Lee, J.-S. Park, J.-S. Woo, and S.-J. Hwang, "Micronization of cilostazol using supercritical antisolvent (SAS) process: effect of process parameters," Powder Technology, vol. 177, no. 2, pp. 64-70, 2007.

[21] M. Rossmann, A. Braeuer, A. Leipertz, and E. Schluecker, "Manipulating the size, the morphology and the polymorphism of acetaminophen using supercritical antisolvent (SAS) precipitation," Journal of Supercritical Fluids, vol. 82, pp. 230-237, 2013.

[22] M. A. Presta, B. Bruyneel, R. Zanella, J. Kool, J. G. Krabbe, and H. Lingeman, "Determination of flavonoids and resveratrol in wine by turbulent-flow chromatography-LC-MS," Chromatographia, vol. 69, no. 2, pp. 167-173, 2009.

[23] V. Glavnik, B. Simonovska, A. Albreht, and I. Vovk, "TLC and HPLC screening of p-coumaric acid, trans-resveratrol, and pterostilbene in bacterial cultures, food supplements, and wine," Journal of Planar Chromatography-Modern TLC, vol. 25, no. 3, pp. 251-258, 2012.

[24] J. Hu, W. K. Ng, Y. C. Dong, S. C. Shen, and R. B. H. Tan, "Continuous and scalable process for water-redispersible nanoformulation of poorly aqueous soluble APIs by antisolvent precipitation and spray-drying," International Journal of Pharmaceutics, vol. 404, no. 1-2, pp. 198-204, 2011.

[25] J.-S. Kim, M.-S. Kim, H. J. Park, S.-J. Jin, S. Lee, and S.-J. Hwang, "Physicochemical properties and oral bioavailability of 
amorphous atorvastatin hemi-calcium using spray-drying and SAS process," International Journal of Pharmaceutics, vol. 359, no. 1-2, pp. 211-219, 2008.

[26] A. Amri, J. C. Chaumeil, S. Sfar, and C. Charrueau, "Administration of resveratrol: what formulation solutions to bioavailability limitations?" Journal of Controlled Release, vol. 158, no. 2, pp. 182-193, 2012.

[27] E. Aghemenloh, J. O. Umukoro, S. O. Azi, S. Yusuf, and J. O. A. Idiodi, "Surface energy calculation of bcc metals using the analytical equivalent crystal theory method," Computational Materials Science, vol. 50, no. 12, pp. 3290-3296, 2011.

[28] D. R. Ely, R. E. García, and M. Thommes, "Ostwald-Freundlich diffusion-limited dissolution kinetics of nanoparticles," Powder Technology, vol. 257, no. 5, pp. 120-123, 2014.

[29] F. Kesisoglou, S. Panmai, and Y. Wu, "Nanosizing - Oral formulation development and biopharmaceutical evaluation," Advanced Drug Delivery Reviews, vol. 59, no. 7, pp. 631-644, 2007.

[30] Y. Hattori, Y. Haruna, and M. Otsuka, "Dissolution process analysis using model-free Noyes-Whitney integral equation," Colloids and Surfaces B: Biointerfaces, vol. 102, no. 1, pp. 227-231, 2013.

[31] M. I. Cabrera, J. A. Luna, and R. J. A. Grau, "Modeling of dissolution-diffusion controlled drug release from planar polymeric systems with finite dissolution rate and arbitrary drug loading," Journal of Membrane Science, vol. 280, no. 1-2, pp. 693-704, 2006.

[32] C. Galli, "Experimental determination of the diffusion boundary layer width of micron and submicron particles," International Journal of Pharmaceutics, vol. 313, no. 1-2, pp. 114-122, 2006. 

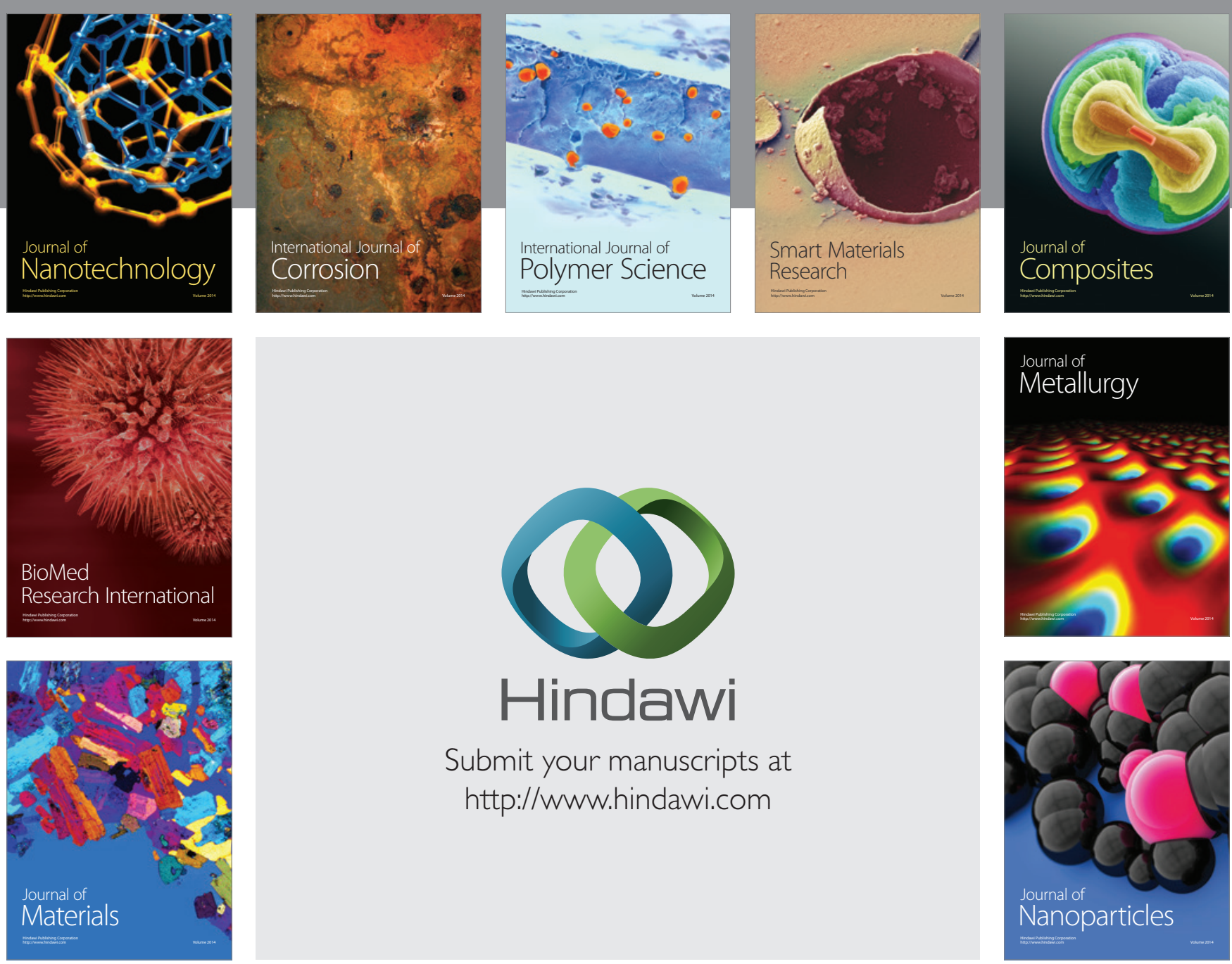

Submit your manuscripts at http://www.hindawi.com
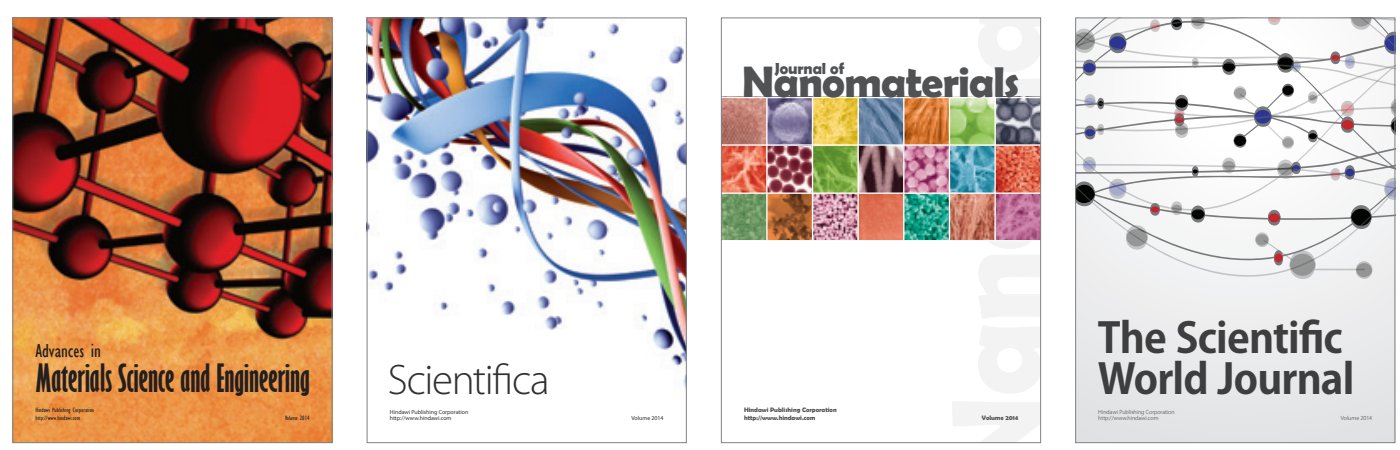

\section{The Scientific World Journal}
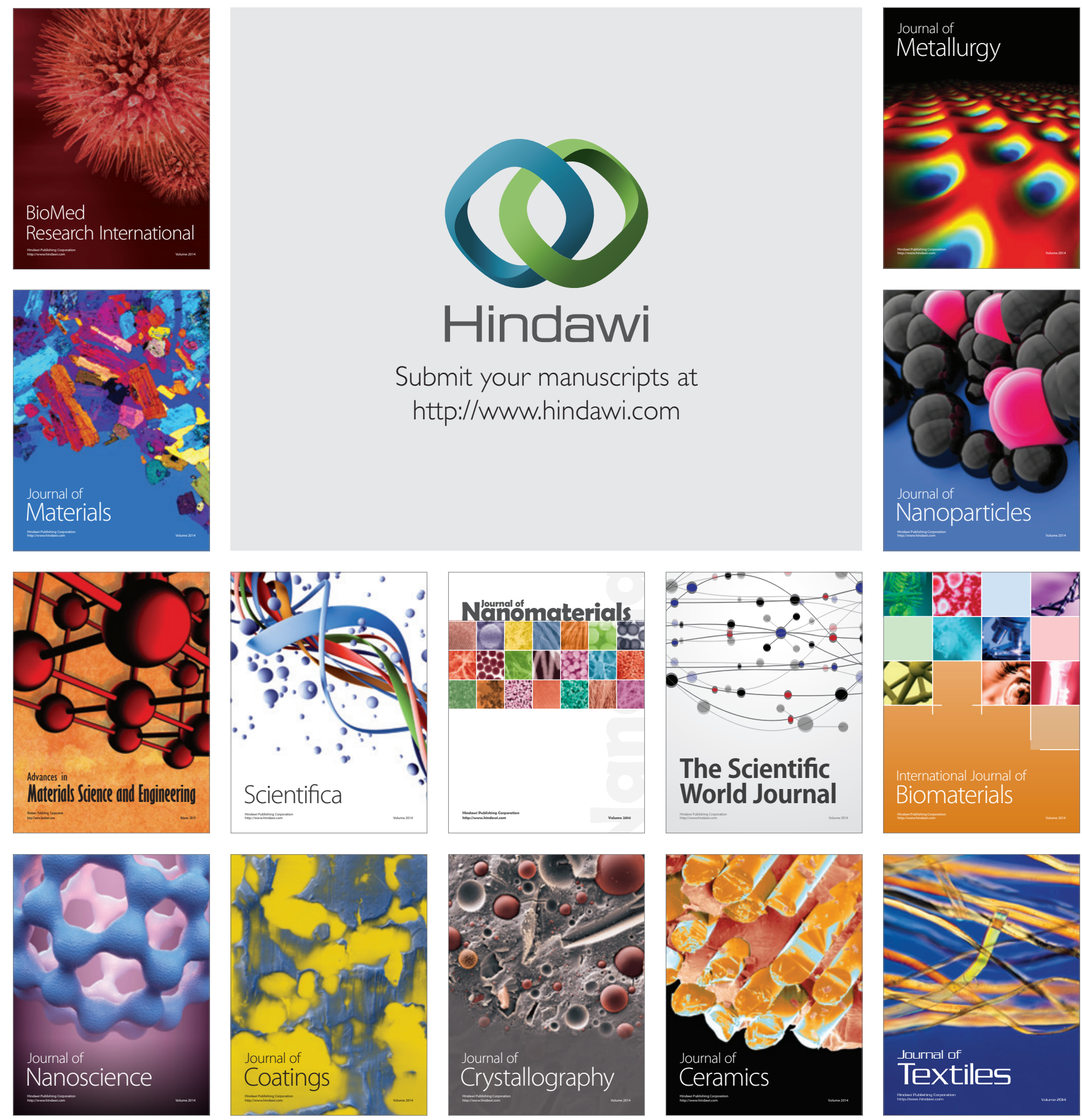